\title{
CLONES PROMISSORES DE SERINGUEIRA PARA A REGIÃO DO VALE DO RIBEIRA, SÃO PAULO' 1
}

\author{
PAULO DE SOUZA GONÇALVES ${ }^{2}$, LUIZ ALBERTO SAES ${ }^{3}$, EDSON LUIZ FURTADO ${ }^{4}$, \\ ROSANA SAMBUGARO ${ }^{5}$ e MAURO SAKAI ${ }^{6}$
}

\begin{abstract}
RESUMO - Este trabalho objetivou apresentar resultados da primeira seleção de clones de seringueira (Hevea brasiliensis (Willd. ex Adr. de Juss.) Muell.-Arg.) da série IAC 300, e amazônicos das séries IAN e Fx, em experimento de pequena escala, visando produção, crescimento e resistência ao mal-dasfolhas. A produção e o vigor de 20 clones foram avaliados por dois e nove anos, respectivamente, em Pariqüera-Açú, no Vale do Ribeira, SP. Os clones IAC 301, IAC 304, IAC 306 e IAC 319 produziram satisfatoriamente nos dois primeiros anos de sangria. Os clones amazônicos IAN 6323, Fx 3864 e IAN 2903, com produções de $1.078 \mathrm{~kg}, 945 \mathrm{~kg}$ e $900 \mathrm{~kg} / \mathrm{ha} / \mathrm{ano}$, respectivamente, foram superiores à testemunha IAN 873 ( $878 \mathrm{~kg} / \mathrm{ha} / \mathrm{ano})$. Os clones selecionados apresentaram crescimento vigoroso, com extremos de perímetro do caule, na abertura do painel, de 37,40 cm (IAN 4493) a 53,75 cm (IAN 6323), e percentual de plantas aptas à sangria de 7,0\% (IAN 4493) a 100\% (IAN 6323 e IAC 302), exceto os clones Fx 3899 e IAN 3044. O IAC 315, com 7,37 mm, mostrou maior espessura de casca virgem que o IAN $873(6,44 \mathrm{~mm})$. Os clones IAC 320, IAC 306 e IAC 315 foram os mais resistentes ao Microcyclus ulei.
\end{abstract}

Termos para indexação: Hevea brasiliensis, crescimento, rendimento, sangria, resistência a pragas.

\section{PROMISING HEVEA CLONES FOR RIBEIRA RIVER VALLEY REGION, SÃO PAULO STATE, BRAZIL}

\begin{abstract}
The objective of this paper was to show first selection results of IAC 300 and Amazonian IAN and Fx series of rubber tree (Hevea brasiliensis (Willd. ex Adr. de Juss) Muell.-Arg.) clones in small scale trial evaluation, for yield, growth and SALB performance. Yield and vigour of 20 clones were assessed for two and nine years, respectively, in Vale do Ribeira, SP, Brazil. In the IAC 300 serie, four clones (IAC 301, IAC 304, IAC 306 and IAC 319) were classified as satisfactory in yielding. The Amazonian clones IAN 6323, Fx 3864 and IAN 2903 produced 1,078 kg, $945 \mathrm{~kg}$ and $900 \mathrm{~kg} / \mathrm{ha} /$ year, respectively, higher than the control IAN 873 (878 kg/ha/year). All selected clones had vigorous growing, with the girth at opening $(\mathrm{cm})$ ranging from 37.40 (IAN 4493) to 53.75 (IAN 6323) and tappability ranging from 7.0\% (IAN 4493) to 100\% (IAC 6323 and IAC 302), except for Fx 3899 and IAN 3044. The IAC 315, with $7.37 \mathrm{~mm}$, had bigger thickness virgin bark than IAN $873(6.44 \mathrm{~mm})$. Concerned to SALB performance, clones IAC 306, IAC 315, IAC 320 were the best in resistance.
\end{abstract}

Index terms: Hevea brasiliensis, growth, yields, tapping, pest resistance.

\footnotetext{
${ }^{1}$ Aceito para publicação em 9 de fevereiro de 2000. Parcialmente financiada pela FAPESP.

${ }^{2}$ Eng. Agrôn., Dr., Embrapa/IAC, Programa Integrado de São Paulo, Programa Seringueira do Centro Café e Plantas Tropicais, Instituto Agronômico (IAC), Caixa Postal 28, CEP 13001-970 Campinas, SP. E-mail: paulog@cec.iac.br

${ }^{3}$ Eng. Agrôn., M.Sc., Núcleo de Agronomia do Vale do Ribeira, IAC, Caixa Postal 122, CEP 11900-000 Registro, SP. E-mail: enar@rgt.matrix.com.br
}

\footnotetext{
${ }^{4}$ Eng. Agrôn., Dr., Prof. Assistente, Dep. de Defesa Fitossanitária, Faculdade de Ciências Agronômicas, UNESP/ Botucatu, Caixa Postal 237, CEP 18603-970 Botucatu, SP. E-mail: elfurtado@fca.unesp.br

${ }^{5}$ Eng. Florestal, Programa Seringueira, Centro de Café e Plantas Tropicais, IAC, Campinas, SP. Bolsista da FAPESP. E-mail: rosanasambugaro@mailcity.com

${ }^{6}$ Eng. Agrôn., Dr., Núcleo de Agronomia do Vale do Ribeira, IAC. E-mail: enar@rgt.matrix.com.br
} 


\section{INTRODUÇÃO}

Os trabalhos de pesquisa com seringueira (Hevea brasiliensis (Willd. ex-Adr. de Juss) Muell.- Arg) conduzidos pelo Instituto Agronômico (IAC), SP, foram iniciados a partir de 1952, quando foram importadas da Companhia Firestone, na Libéria (África), sementes ilegítimas de Tjir 1 x Tjir 16. Além de servirem para atestar a viabilidade do cultivo da seringueira no Estado, foram submetidas ao controle de produção, originando os primeiros clones para fins de recomendação em larga escala. Ao mesmo tempo em que se procedia à importação de sementes da Libéria, o IAC providenciava a introdução, do Sudeste asiático e do Instituto Agronômico do Norte (IAN), de dezenas de clones de alta produção. Tais introduções permitiram, além da formação de seringais, o estabelecimento de coleções para o início do melhoramento genético, permitindo, assim, a obtenção de dados de produção de alguns clones que haviam sido plantados em diferentes áreas do Estado (Cunha, 1966). De outras regiões, importou clones amazônicos com diferentes níveis de resistência ao mal-das-folhas causado pelo Microcyclus ulei (P. Henn.) v. Arx, encontrando-se disponíveis ao programa de melhoramento genético.

Considerando-se que o Estado de São Paulo possui duas regiões ecologicamente aptas ao cultivo dessa espécie, ou seja, a região do Planalto e a região Litorânea, foi em 1965 que o programa de melhoramento do IAC teve início, com objetivo de obter clones de alta produção para a região do Planalto e clones resistentes e produtivos para a região do Litoral do Estado. A dualidade do programa levou em consideração o fato de que o Planalto Paulista não tem mostrado problemas de ataques epidêmicos do M. ulei. Já o litoral, por suas condições ecológicas, favorece o aparecimento de ataques severos desse patógeno.

Segundo Gonçalves et al. (1988), foi a partir de 1987 que o programa tomou impulso, dando-se ênfase não somente à produção e à resistência a doenças mas também aos atributos secundários, como, por exemplo, resistência ao vento e formato da copa. Desde então, o IAC vem avaliando cerca de 700 novos clones em experimentos de pequena escala, instalados nas unidades experimentais do Planalto, em
Votuporanga, Pindorama e Jaú, e no litoral, em Pariqüera-Açú (Vale do Ribeira).

Este trabalho apresenta resultados de produção e alguns caracteres secundários de clones da nova série IAC 300 e alguns clones amazônicos resultantes da primeira seleção com potencial para plantio no litoral e no Vale do Ribeira, no Estado de São Paulo.

\section{MATERIAL E MÉTODOS}

O experimento foi instalado em janeiro de 1989, no Núcleo de Agronomia do Vale do Ribeira, do Instituto Agronômico de Campinas (IAC), em Pariqüera-Açú a $24^{\circ} 43^{\prime} \mathrm{S}$ de latitude, $47^{\circ} 57^{\prime} \mathrm{W}$ de longitude e $25 \mathrm{~m}$ de altitude, em solo Podzólico Vermelho-Amarelo "integrade" para Latossolo Vermelho-Amarelo, de textura argilosa, profundo, topografia ligeiramente ondulada e bem drenado (Sakai et al., 1983).

Predomina, nessa área, o clima tropical úmido Af (Köppen) com transição para Cfa, sem estação seca definida. A temperatura média anual é de $20,8^{\circ} \mathrm{C}$, umidade relativa de $85,0 \%$ e pluviosidade média anual de $1.517 \mathrm{~mm}$, com predominância de chuvas elevadas de outubro a abril e mais reduzidas de maio a setembro.

Os clones utilizados foram sete amazônicos e treze da série IAC 300, cujos paternais encontram-se na Tabela 1. Os clones IAC são resultantes de polinizações controladas, efetuadas no período de 1985 a 1988. As sementes obtidas foram plantadas em viveiros de cruzamentos estabelecidos nas Estações Experimentais de Pindorama e Pariqüera-Açú nos anos subseqüentes às polinizações, no espaçamento de $1,0 \mathrm{~m} \times 1,0 \mathrm{~m}$, agrupadas em progênies. Aos 48 meses as plantas foram submetidas à seleção com base em produção (Teste HMM), vigor, e em seguida decepadas, objetivando a produção de hastes rejuvenescidas.

Os clones amazônicos foram recebidos da EmbrapaCentro de Pesquisa Agroflorestal da Amazônia Ocidental, através de introduções efetuadas no período de 1980 a 1983 e instaladas na coleção de clones da antiga Seção de Plantas Tropicais do IAC, situada no Núcleo Experimental de Campinas (NEC). Dois anos antes da instalação do experimento, os clones multiplicados no jardim clonal foram submetidos à seleção com base no vigor. As hastes produzidas dos clones IAC e amazônicos foram enxertadas pelo método convencional, em que as gemas maduras dos clones em estudo foram enxertadas em porta-enxertos ensacados com 12 a 14 meses de idade, oriundos de sementes de polinização aberta de uma população de pés-francos.

$\mathrm{Na}$ instalação do experimento no campo, adotou-se o delineamento em blocos ao acaso, com três repetições, 
TABELA 1. Paternais de 20 clones de seringueira selecionados no Experimento de Avaliação de Clones em Pequena Escala, instalado no Núcleo de Agronomia do Vale do Ribeira, Pariqüera-Açú, SP1.

\begin{tabular}{|c|c|}
\hline Clones & Paternais \\
\hline IAC 300 & RRIM 605 (Tjir 1 x PB 49) x AVROS 353 \\
\hline IAC 301 & RRIM 501 (Pil A 44 x Lun) x AVROS 1518 (AVROS 214 x AVROS 256) \\
\hline IAC 302 & RRIM 501 (Pil A 44 x Lun) x AVROS 353 \\
\hline IAC 304 & RRIM 605 (Tjir 1 x PB 86) x AVROS 1518 (AVROS 214 x AVROS 256) \\
\hline IAC 306 & AVROS 49 x RRIM 507 (Pil B 84 x Pil A 44) \\
\hline IAC 315 & AVROS 1328 x AVROS 49 \\
\hline IAC 319 & Tjir 1 x Fx 505 (F 4542 x AVROS 363) \\
\hline IAC 320 & GT 127 x Fx $652($ F 4542 x Tjir 1) \\
\hline IAC 323 & AVROS 1126 x Fx 25 (F 351 x AVROS 49) \\
\hline IAC 324 & AVROS 1126 x Fx 4098 (PB 86 x FB 110) \\
\hline IAC 326 & RRIM 623 (PB 49 x Pil B 84) x Fx 25 (F 351 x AVROS 49) \\
\hline IAC 327 & RRIM 605 (Tjir 1 x PB 86) x RRIM 600 (Tjir 1 x PB 86) \\
\hline IAC 328 & RRIM 600 (Tjir 1 x PB 86) x PR 107 \\
\hline Fx 3864 & PB 86 x FB 38 \\
\hline Fx 3899 & F $4542 \times$ AVROS 363 \\
\hline IAN 2903 & Fx 516 (F $4542 \times$ AVROS 363) x PB 86 \\
\hline IAN 3044 & Fx 25 (F 351 x AVROS 49) x RRIM 600 (Tjir 1 x PB 86) \\
\hline IAN 4493 & Fx 4421 (F 4573 x PB 86) x Tjir 1 \\
\hline IAN 6323 & Tjir 1 x Fx 3810 (F 4542 x AVROS 363) \\
\hline IAN 873 & PB 86 x FA 1717 \\
\hline
\end{tabular}

1 Fx: Cruzamento Ford; IAN: Instituto Agronômico do Norte; IAC: Instituto Agronômico de Campinas; F: Ford; AVROS: Algemene Vereniging Rubber Planters Oostkust Sumatra; PB: Prang Besar; FB: Ford Belem; Fx: Ford (cruzamentos); RRIM: Rubber Research Institute of Malaysia; Tjir: Tjirandji; Pil: Pilmoor; Lun: Lunderston; GT: Gondang Tapen; FA: Ford Acre; Gl: Glenshiel; PR: Proefstation voor Rubber.

sendo cada clone representado por seis plantas, em parcela linear, plantadas no espaçamento de 7,0 m entre linhas e 3,0 m entre plantas.

Durante o período de avaliação do vigor dos clones, mensurações anuais do perímetro do caule foram feitas a $0,50 \mathrm{~m}$ acima do calo de enxertia, no primeiro ano, e a 1,20 m, a partir do segundo ano.

$\mathrm{Na}$ avaliação da produção dos clones a partir do sétimo ano, foram utilizados os sistemas de sangria, $1 / 2 \mathrm{~S} \mathrm{~d} / 25 \mathrm{~d} / 7$ no primeiro ano, e $1 / 2 \mathrm{~S} \mathrm{~d} / 3 \mathrm{dd} / 7$. ET $2,5 \% \mathrm{~Pa}$. 10/y no segundo ano, tendo o clone IAN 873 como testemunha.

O registro da produção foi efetuado pelo látex coagulado nas tigelas, coletado ao acaso duas vezes ao mês, secado em condições normais de sombra e ventilação ao longo do período de avaliação, preso a cada árvore sangrada. O total anual de borracha por árvore foi então dividido pelo número de "coágulos", sendo o resultado expresso em gramas/ árvore/sangria. A média geral em gramas/árvore/sangria foi calculada para gramas/árvore/clone/ano. A partir desses dados, estimou-se a produção por clone/hectare/ano, e, segundo recomendações da Embrapa (1989), adotaram-se 240 árvores no primeiro ano e 340 no segundo, o que corresponde a $60 \%$ e $85 \%$, respectivamente, de um estande de 400 plantas por hectare. Foram consideradas, no siste- ma d/2, 140 sangrias, e no d/3, 104 sangrias/ano (Abraham, 1992).

Amostras de casca virgem para determinação da espessura e número de anéis de vasos laticíferos foram tomadas a 1,0 m de altura do caule de cada árvore, com o auxílio de um "vazador". A espessura da casca foi determinada com paquímetro, e o número de anéis de vasos laticíferos foi obtido por meio de secções longitudinais radiais da casca.

O mal-das-folhas foi quantificado diretamente nas brotações - em dois estádios de desenvolvimento dos folíolos -, denominadas brotações novas e maduras e apresentando as fases conidiais e ascógenas do patógeno, respectivamente, conforme escala diagramática proposta por Chee (1976), em duas épocas de avaliação (janeiro e abril). Avaliou-se o desfolhamento natural das plantas, no ano de 1996, visando verificar o hábito decíduo e também o desfolhamento secundário, provocado pelo M. ulei, conforme escala proposta por Gomes et al. (1983), modificada por Furtado (1990). A antracnose, que ocorreu apenas no ano de 1995, numa escala endêmica, foi avaliada de acordo com a escala proposta por Wastie (1973).

Durante a execução do experimento, empregaram-se todas as práticas culturais convencionais ao cultivo da seringueira (Cardoso, 1982). 


\section{RESULTADOS E DISCUSSÃO}

Na Tabela 2 são apresentados dados de produção de borracha seca em gramas/árvore/sangria, estimativas de produção de borracha seca/hectare/ano e porcentagem de produção em relação à testemunha (IAN 873). Considerando cerca de 240 árvores por hectare daquelas aptas à sangria no primeiro ano, sob o sistema $1 / 2 \mathrm{~S} \mathrm{~d} / 35 \mathrm{~d} / 7$, observou-se que os clones IAN 2903, IAN 4493 e IAN 6323 foram os que

TABELA 2. Produção média anual de borracha (kg/ha/ano) e, entre parênteses, produção de borracha/sangria/árvore (g), de 20 clones selecionados no Vale do Ribeira, Pariqüera-Açú, SP.

\begin{tabular}{|c|c|c|c|c|c|}
\hline Clones & 10 ano $^{1}$ & $2^{\circ} \mathrm{ano}^{2}$ & Total & Média & Porcentagem em relação à testemunha \\
\hline IAC 300 & $\begin{array}{c}462 \\
(13,75)\end{array}$ & $\begin{array}{c}808 \\
(22,86)\end{array}$ & $\begin{array}{c}1270 \\
(36,61)\end{array}$ & $\begin{array}{c}635 \\
(18,31)\end{array}$ & 72 \\
\hline IAC 301 & $\begin{array}{c}534 \\
(15,90)\end{array}$ & $\begin{array}{c}1244 \\
(35,18)\end{array}$ & $\begin{array}{c}1778 \\
(51,08)\end{array}$ & $\begin{array}{c}889 \\
(25,54)\end{array}$ & 101 \\
\hline IAC 302 & $\begin{array}{c}307 \\
(9,15)\end{array}$ & $\begin{array}{c}653 \\
(18,47)\end{array}$ & $\begin{array}{c}960 \\
(27,62)\end{array}$ & $\begin{array}{c}480 \\
(13,81)\end{array}$ & 55 \\
\hline IAC 304 & $\begin{array}{c}991 \\
(29,50)\end{array}$ & $\begin{array}{c}992 \\
(28,07)\end{array}$ & $\begin{array}{c}1983 \\
(57,57)\end{array}$ & $\begin{array}{c}992 \\
(28,79)\end{array}$ & 113 \\
\hline IAC 306 & $\begin{array}{c}506 \\
(15,07)\end{array}$ & $\begin{array}{c}939 \\
(26,57)\end{array}$ & $\begin{array}{c}1445 \\
(41,64)\end{array}$ & $\begin{array}{c}723 \\
(20,82)\end{array}$ & 82 \\
\hline IAC 315 & $\begin{array}{c}408 \\
(12,14)\end{array}$ & $\begin{array}{c}697 \\
(19,72)\end{array}$ & $\begin{array}{c}1105 \\
(31,86)\end{array}$ & $\begin{array}{c}553 \\
(15,93)\end{array}$ & 63 \\
\hline IAC 319 & $\begin{array}{c}609 \\
(18,12)\end{array}$ & $\begin{array}{c}981 \\
(27,74)\end{array}$ & $\begin{array}{c}1590 \\
(45,86)\end{array}$ & $\begin{array}{c}795 \\
(22,93)\end{array}$ & 91 \\
\hline IAC 320 & $\begin{array}{c}88 \\
(2,61)\end{array}$ & $\begin{array}{c}890 \\
(25,16)\end{array}$ & $\begin{array}{c}978 \\
(27,77)\end{array}$ & $\begin{array}{c}489 \\
(13,89)\end{array}$ & 56 \\
\hline IAC 323 & $\begin{array}{c}148 \\
(4,40)\end{array}$ & $\begin{array}{c}799 \\
(22,60)\end{array}$ & $\begin{array}{c}947 \\
(27,00)\end{array}$ & $\begin{array}{c}474 \\
(13,50)\end{array}$ & 54 \\
\hline IAC 324 & $\begin{array}{c}178 \\
(5,29)\end{array}$ & $\begin{array}{c}742 \\
(20,99)\end{array}$ & $\begin{array}{c}920 \\
(26,28)\end{array}$ & $\begin{array}{c}460 \\
(13,14)\end{array}$ & 52 \\
\hline IAC 326 & $\begin{array}{c}248 \\
(7,38)\end{array}$ & $\begin{array}{c}635 \\
(17,96)\end{array}$ & $\begin{array}{c}883 \\
(25,34)\end{array}$ & $\begin{array}{c}442 \\
(12,67)\end{array}$ & 50 \\
\hline IAC 327 & $\begin{array}{c}406 \\
(12,08)\end{array}$ & $\begin{array}{c}679 \\
(19,20)\end{array}$ & $\begin{array}{c}1085 \\
(31,28)\end{array}$ & $\begin{array}{c}543 \\
(15,64)\end{array}$ & 62 \\
\hline IAC 328 & $\begin{array}{c}446 \\
(13,28)\end{array}$ & $\begin{array}{c}747 \\
(21,13)\end{array}$ & $\begin{array}{c}1193 \\
(34,41)\end{array}$ & $\begin{array}{c}597 \\
(17,21)\end{array}$ & 68 \\
\hline Fx 3864 & $\begin{array}{c}499 \\
(14,86)\end{array}$ & $\begin{array}{c}1391 \\
(39,33)\end{array}$ & $\begin{array}{c}1890 \\
(54,19)\end{array}$ & $\begin{array}{c}945 \\
(27,10)\end{array}$ & 108 \\
\hline Fx 3899 & - & $\begin{array}{c}1400 \\
(39,60)\end{array}$ & $\begin{array}{c}1400 \\
(39,60)\end{array}$ & $\begin{array}{c}1400 \\
(39,60)\end{array}$ & 80 \\
\hline IAN 2903 & $\begin{array}{c}698 \\
(20,77)\end{array}$ & $\begin{array}{c}1102 \\
(31,16)\end{array}$ & $\begin{array}{c}1800 \\
(51,93)\end{array}$ & $\begin{array}{c}900 \\
(25,97)\end{array}$ & 103 \\
\hline IAN 3044 & - & $\begin{array}{c}871 \\
(24,62)\end{array}$ & $\begin{array}{c}871 \\
(24,62)\end{array}$ & $\begin{array}{c}871 \\
(24,62)\end{array}$ & 50 \\
\hline IAN 4493 & $\begin{array}{c}574 \\
(17,09)\end{array}$ & $\begin{array}{c}735 \\
(20,80)\end{array}$ & $\begin{array}{c}1309 \\
(37,89)\end{array}$ & $\begin{array}{c}655 \\
(18,95)\end{array}$ & 75 \\
\hline IAN 6323 & $\begin{array}{c}563 \\
(16,75)\end{array}$ & $\begin{array}{c}1592 \\
(45,03)\end{array}$ & $\begin{array}{c}2155 \\
(61,78)\end{array}$ & $\begin{array}{c}1078 \\
(30,89)\end{array}$ & 123 \\
\hline IAN $873^{3}$ & $\begin{array}{c}509 \\
(15,15)\end{array}$ & $\begin{array}{c}1246 \\
(35,25)\end{array}$ & $\begin{array}{c}1755 \\
(50,40)\end{array}$ & $\begin{array}{c}878 \\
(25,20) \\
\end{array}$ & 100 \\
\hline
\end{tabular}

1 Sangria no sistema $1 / 2 \mathrm{~S} \mathrm{~d} / 3$ 5d/7: 140 sangrias/ano, 240 árvores.

2 Sangria no sistema 1/2 S d/3 5d/7: ET 2,5\% Pa.10/y: 104 sangrias/ano, 340 árvores.

3 Testemunha. 
mais produziram no primeiro ano de sangria com 20,77 g, 17,09 g e 16,75 g, respectivamente. Em relação aos clones da série IAC, observou-se que o IAC 304, IAC 319 e IAC 301 foram os mais produtivos, com $29,50,18,12$ e $15,90 \mathrm{~g}$, respectivamente.

No segundo ano, com o emprego do sistema de sangria $1 / 2 \mathrm{~S} \mathrm{~d} / 3 \mathrm{dd} / 7$. ET 2,5\% Pa. 10/y, houve um aumento visível na produção da maioria dos clones, decorrente da estimulação. Resultados da Tabela 2 evidenciam que o clone IAN 6323 foi o que mais produziu, seguido do Fx 3899 e do Fx 3864, que produziram 45,03, 39,60 e 39,33 g, respectivamente, todos superiores ao IAN 873 (35,25 g), considerado um dos clones mais plantados no Vale do Ribeira e no litoral do Estado.

Comparando-se com os dados obtidos na região noroeste do Estado (Gonçalves, 2000), as produções dos clones altamente produtivos IAC 300, IAC 301, IAC 302 encontram-se pouco abaixo da expectativa. Uma das hipóteses para a redução da produção pode ser atribuída à incidência de doenças foliares muito comuns no Vale do Ribeira.

O clone IAC 304 apresentou a mesma produção nos dois anos de avaliação, mesmo utilizando o sistema de sangria na ausência e presença de estimulante (Tabela 2).Uma das hipóteses para essa mesma produção pode ser atribuída ao fato de o clone não reagir à presença de estimulante. Segundo Paardekooper (1989), a resposta de um clone à estimulação depende de certos fatores, alguns dos quais são inerentes à árvore, ao passo que outros fatores podem ser controlados. De acordo com Paardekooper (1989), clones altamente produtivos mostram, geralmente, baixa resposta à estimulação. Outra hipótese é que determinados clones adaptamse diferentemente em ambientes edafoclimaticamente distintos.

A produção de borracha seca dos dois anos relativos aos clones IAC 301, IAC 304, IAN 2903, IAN 6323 e Fx 3864 destacou-se em relação à testemunha, IAN 873 (Tabela 2).

A produção média dos dois primeiros anos de sangria (Tabela 2) foi maior no clone IAN 6323, com $1.078 \mathrm{~kg} / \mathrm{ha} /$ ano de borracha seca, superior em relação ao IAN 873 em 23\%, seguido dos clones IAC 304 e Fx 3864, que produziram $992 \mathrm{~kg} / \mathrm{ha} / \mathrm{ano}$ e $945 \mathrm{~kg} / \mathrm{ha} / \mathrm{ano}, 13 \%$ e $8 \%$ superiores ao IAN 873, res- pectivamente. Exceto o IAN 2903 e o IAC 301, os demais apresentaram produções médias inferiores às do IAN 873.

As médias de perímetro anual do caule, no que diz respeito aos períodos de imaturidade e adulto de cada clone são apresentadas na Tabela 3 . O desempenho de crescimento dos clones IAC 324, IAC 315, IAC 328, IAC 323, Fx 3864 e IAN 6323 foi superior ao da testemunha. Pode-se verificar que o modelo de crescimento variou de clone para clone, ou seja: IAC 315, IAC 328, Fx 3864, IAC 324, IAC 323 e IAN 6323 alcançaram médias superiores a $45 \mathrm{~cm}$ de perímetro do caule aos seis anos de idade, enquanto o IAN 873 alcançou essa média no sétimo ano de idade. Os clones IAC 301, IAC 304 e IAC 327, juntamente com os amazônicos Fx 3899, IAN 3044 e IAN 4493, mostraram o mais longo período de imaturidade, pois alcançaram somente aos oito e nove anos de idade a média de perímetro do caule necessária para abertura do painel. O clone IAN 6323, que ocupava a primeira posição de clone mais vigoroso no sétimo ano de crescimento (abertura do painel), foi superado pelos Fx 3864, IAC 324 e IAC 315 no nono ano de crescimento, ou seja, no segundo ano de sangria. Esse fato pode ser explicado pelo atraso do seu crescimento após o procedimento da sangria. Segundo Tan (1987), clones altamente produtivos apresentam, de modo geral, crescimento lento durante o período de explotação do látex. Nesta fase, o fotossintetizado é repartido em duas fontes competidoras: látex explotado e crescimento do caule. Segundo Wycherley (1976), em alguns clones a maior parte do caule é direcionada para o látex explotado, e apresenta crescimento lento, tornando-se, muitas vezes, favorável à quebra pelo vento.

A Tabela 4 mostra o incremento médio anual do caule, referente aos períodos de imaturidade e adulto, relativos aos nove anos de crescimento dos 20 clones. A maior média anual no período de imaturidade foi observada no IAN 6323, com 7,68 cm, seguido pelos clones Fx 3864, IAC 324 e IAC 315, estes com médias de 7,46 cm, 7,35 cm e 7,34 cm, respectivamente, todas superiores à do IAN $873 \mathrm{com}$ $6,86 \mathrm{~cm}$.

A maior média de incremento anual para a maioria dos clones selecionados foi no terceiro para o quarto ano, com média de $10,99 \mathrm{~cm}$ (Tabela 4). O maior 
TABELA 3. Perímetro médio anual do caule $(\mathrm{cm})$ referente ao período de imaturidade e adulto de 20 clones de seringueira, selecionados no Vale do Ribeira/IAC, Pariqüera-Açú, SP.

\begin{tabular}{|c|c|c|c|c|c|c|c|c|c|c|}
\hline \multirow[t]{2}{*}{ Clones } & \multirow{2}{*}{$\begin{array}{c}\mathrm{N}^{\mathrm{o}} \mathrm{de} \\
\text { plantas }\end{array}$} & \multicolumn{7}{|c|}{ Período de imaturidade $^{1}$} & \multicolumn{2}{|c|}{ Período adulto $^{2}$} \\
\hline & & $1^{\mathrm{o}} \mathrm{ano}^{3}$ & $2^{-}$ano $^{4}$ & $3^{-}$ano & $4^{\mathrm{o}}$ ano & $5^{\circ}$ ano & $6^{-}$ano & $7^{\mathrm{o}}$ ano $^{5}$ & $8^{\mathrm{o}}$ ano & $9^{-}$ano \\
\hline IAC 300 & 17 & 5,24 & 8,89 & 13,39 & 24,75 & 33,35 & 40,44 & 43,15 & 47,44 & 49,59 \\
\hline IAC 301 & 17 & 5,24 & 9,18 & 14,86 & 23,31 & 32,89 & 38,31 & 39,59 & 41,75 & 43,82 \\
\hline IAC 302 & 15 & 7,32 & 8,92 & 16,10 & 26,58 & 39,11 & 43,41 & 47,64 & 48,33 & 53,09 \\
\hline IAC 304 & 9 & 5,65 & 9,64 & 14,22 & 25,43 & 32,50 & 39,86 & 43,27 & 44,00 & 45,27 \\
\hline IAC 306 & 18 & 8,01 & 12,19 & 17,44 & 30,65 & 37,50 & 44,32 & 48,68 & 53,65 & 54,89 \\
\hline IAC 315 & 17 & 7,30 & 10,53 & 16,08 & 31,88 & 41,13 & 47,93 & 51,41 & 54,59 & 56,28 \\
\hline IAC 319 & 13 & 8,44 & 12,23 & 16,00 & 27,57 & 37,31 & 45,55 & 47,04 & 51,77 & 53,92 \\
\hline IAC 320 & 10 & 9,11 & 12,04 & 18,00 & 29,71 & 36,29 & 45,67 & 46,98 & 47,40 & 48,36 \\
\hline IAC 323 & 10 & 6,65 & 10,28 & 16,78 & 30,00 & 44,33 & 46,13 & 49,75 & 52,10 & 54,75 \\
\hline IAC 324 & 8 & 6,96 & 11,69 & 18,13 & 33,63 & 44,81 & 49,31 & 51,46 & 57,63 & 62,75 \\
\hline IAC 326 & 11 & 5,13 & 7,46 & 11,69 & 25,50 & 34,25 & 44,36 & 46,16 & 48,06 & 48,91 \\
\hline IAC 327 & 14 & 6,18 & 8,67 & 14,92 & 23,43 & 30,29 & 35,75 & 37,75 & 40,07 & 42,71 \\
\hline Fx 3864 & 11 & 7,55 & 10,63 & 19,07 & 34,30 & 43,17 & 49,89 & 52,20 & 56,80 & 57,27 \\
\hline Fx 3899 & 17 & 4,56 & 6,72 & 11,40 & 21,75 & 29,31 & 35,38 & 38,74 & 42,44 & 43,65 \\
\hline IAN 2903 & 16 & 6,39 & 8,72 & 18,10 & 19,42 & 26,83 & 33,18 & 38,45 & 41,27 & 44,75 \\
\hline IAN 3044 & 15 & 5,03 & 8,61 & 15,58 & 21,00 & 28,00 & 34,38 & 38,43 & 39,57 & 42,00 \\
\hline IAN 4493 & 18 & 5,79 & 8,50 & 14,50 & 21,13 & 28,90 & 36,04 & 37,40 & 40,27 & 45,17 \\
\hline IAN 6323 & 13 & 6,36 & 8,68 & 15,70 & 29,56 & 40,00 & 47,89 & 53,75 & 54,92 & 56,05 \\
\hline IAN $873^{6}$ & 52 & 8,81 & 9,75 & 16,04 & 29,38 & 38,95 & 44,93 & 47,99 & 52,00 & 53,61 \\
\hline Média geral & & $6,52 \pm 1,39$ & $9,57 \pm 1,58$ & $15,70 \pm 2,03$ & $26,69 \pm 4,34$ & $36,04 \pm 5,64$ & $42,50 \pm 5,37$ & $45,50 \pm 5,46$ & $48,27 \pm 5,96$ & $50,49 \pm 5,86$ \\
\hline
\end{tabular}

${ }^{1} \mathrm{O}$ período de imaturidade é anterior à abertura do painel de sangria.

${ }^{2} \mathrm{O}$ período adulto é posterior à abertura do painel de sangria.

${ }^{3}$ Dados coletados a $0,50 \mathrm{~cm}$ acima do calo de enxertia.

${ }^{4} \mathrm{~A}$ partir do segundo ano os dados foram coletados a 1,20 m acima do calo de enxertia.

${ }^{5}$ Abertura do painel de sangria.

${ }^{6}$ Testemunha. 
TABELA 4. Incremento anual do crescimento do caule $(\mathrm{cm})$ referente ao período de imaturidade e adulto de 20 clones de seringueira, selecionados no Vale do Ribeira, Pariqüera-Açú, SP.

\begin{tabular}{|c|c|c|c|c|c|c|c|c|c|c|c|c|}
\hline \multirow[t]{2}{*}{ Clones } & \multirow{2}{*}{$\begin{array}{l}\mathrm{N}^{\mathrm{o}} \mathrm{de} \\
\text { plantas }\end{array}$} & \multicolumn{8}{|c|}{ Período de imaturidade $^{1}$} & \multicolumn{3}{|c|}{ Período adulto $^{2}$} \\
\hline & & $0-1$ & $1-2$ & $2-3$ & $3-4$ & $4-5$ & $5-6$ & $6-7$ & Média & $7-8$ & $8-9$ & Média \\
\hline IAC 300 & 17 & 5,24 & 3,65 & 4,50 & 11,36 & 8,60 & 7,09 & 2,71 & 6,16 & 4,29 & 2,15 & 3,22 \\
\hline IAC 301 & 17 & 5,24 & 3,94 & 5,68 & 8,45 & 9,58 & 5,42 & 1,28 & 5,66 & 2,16 & 2,16 & 2,16 \\
\hline IAC 302 & 15 & 7,32 & 1,60 & 7,18 & 10,48 & 12,53 & 4,30 & 4,23 & 6,81 & 0,69 & 4,76 & 2,73 \\
\hline IAC 304 & 9 & 5,65 & 3,99 & 4,58 & 11,21 & 7,07 & 7,36 & 3,41 & 6,18 & 0,73 & 1,27 & 1,00 \\
\hline IAC 306 & 18 & 8,01 & 4,18 & 5,25 & 13,21 & 6,85 & 6,82 & 4,36 & 6,95 & 4,97 & 1,24 & 3,11 \\
\hline IAC 315 & 17 & 7,30 & 3,23 & 5,55 & 15,80 & 9,25 & 6,80 & 3,48 & 7,34 & 3,18 & 1,69 & 2,44 \\
\hline IAC 319 & 13 & 8,44 & 3,79 & 3,77 & 11,57 & 9,74 & 8,24 & 1,49 & 6,72 & 4,73 & 2,15 & 3,44 \\
\hline IAC 320 & 10 & 9,11 & 2,93 & 5,96 & 11,71 & 6,58 & 9,38 & 1,31 & 6,71 & 0,42 & 0,96 & 0,69 \\
\hline IAC 323 & 10 & 6,65 & 3,63 & 6,50 & 13,22 & 14,33 & 1,80 & 3,62 & 7,11 & 2,35 & 2,65 & 2,50 \\
\hline IAC 324 & 8 & 6,96 & 4,73 & 6,44 & 15,50 & 11,18 & 4,50 & 2,15 & 7,35 & 6,17 & 5,12 & 5,65 \\
\hline IAC 326 & 11 & 5,13 & 2,33 & 4,23 & 13,81 & 8,75 & 10,11 & 1,80 & 6,59 & 1,90 & 0,85 & 1,38 \\
\hline IAC 327 & 14 & 6,18 & 2,49 & 6,25 & 8,51 & 6,86 & 5,46 & 2,00 & 5,39 & 2,32 & 2,64 & 2,48 \\
\hline IAC 328 & 6 & 4,63 & 3,51 & 7,86 & 8,86 & 16,97 & 5,39 & 3,00 & 7,17 & 1,11 & 1,67 & 1,39 \\
\hline Fx 3864 & 11 & 7,55 & 3,08 & 8,44 & 15,23 & 8,87 & 6,72 & 2,31 & 7,46 & 4,60 & 0,47 & 2,54 \\
\hline Fx 3899 & 17 & 4,56 & 2,16 & 4,68 & 10,35 & 7,56 & 6,07 & 3,36 & 5,53 & 3,70 & 1,21 & 2,46 \\
\hline IAN 2903 & 16 & 6,39 & 2,33 & 9,38 & 1,32 & 7,41 & 6,35 & 5,27 & 5,49 & 2,82 & 3,48 & 3,15 \\
\hline IAN 3044 & 15 & 5,03 & 3,58 & 6,97 & 5,42 & 7,00 & 6,38 & 4,05 & 5,49 & 1,14 & 2,43 & 1,79 \\
\hline IAN 4493 & 18 & 5,79 & 2,71 & 6,00 & 6,63 & 7,77 & 7,14 & 1,36 & 5,34 & 2,87 & 4,90 & 3,89 \\
\hline IAN 6323 & 13 & 6,36 & 2,32 & 7,02 & 13,86 & 10,44 & 7,89 & 5,86 & 7,68 & 1,17 & 1,13 & 1,15 \\
\hline IAN $873^{3}$ & 52 & 8,81 & 0,94 & 6,29 & 13,34 & 9,57 & 5,98 & 3,06 & 6,86 & 4,01 & 1,61 & 2,81 \\
\hline Média geral & & $6,52 \pm 1,39$ & $3,06 \pm 0,94$ & $6,13 \pm 1,44$ & $10,99 \pm 3,67$ & $9,35 \pm 2,71$ & $6,46 \pm 1,82$ & $3,01 \pm 1,32$ & $6,50 \pm 0,78$ & $2,77 \pm 1,65$ & $2,23 \pm 1,37$ & $2,50 \pm 1,14$ \\
\hline
\end{tabular}


incremento do perímetro do caule nessa idade, de $15,80 \mathrm{~cm}$, foi apresentado pelo IAC 315 , seguido pelos clones IAC 324 e Fx 3864 com valores de $15,50 \mathrm{~cm}$ e 15,23 cm, respectivamente. Os dois clones mais vigorosos (Fx 3864 e IAC 315) mostraram incremento médio anual do perímetro excedendo $7,00 \mathrm{~cm}$ por todo período de imaturidade.

O crescimento dos clones após a abertura do painel foi estimado considerando-se o incremento anual do caule nos dois anos de sangria. O crescimento constante no período de explotação contribui para a redução de quebra de árvores pelo vento. Segundo Abraham \& Tayler (1967), a sangria influi bastante no incremento anual de um clone, mas existem vários clones com alta capacidade de produção e vigor que mantém produção constante durante a fase adulta.

Os incrementos anuais do caule foram maiores na fase imatura, em comparação com o período adulto, o que indica que o crescimento das árvores, na maioria dos clones, é afetado pela explotação do látex (Tabela 4).

A maior média de incremento anual de crescimento no período adulto foi apresentada pelo clone IAC 324, seguido pela dos clones IAN 4493 e IAC 319 , estes com médias $5,65 \mathrm{~cm}, 3,89 \mathrm{~cm} \mathrm{e} 3,44 \mathrm{~cm}$, respectivamente, todos bem superiores à média ge$\operatorname{ral}(2,50 \mathrm{~cm})$ e à do IAN $873(2,81 \mathrm{~cm})$.

As médias de porcentagens de plantas aptas à sangria, o número médio dos anéis de vasos laticíferos e a espessura média da casca virgem são apresentados na Tabela 5.

A média do perímetro do caule das plantas aptas à abertura dos painéis está relacionada com o percentual de plantas aptas à sangria. Esse percentual, contido na Tabela 5, variou de $0 \%$ (Fx 3899 e IAN 3044) a 100\% (IAC 302, IAN 6323). Observou-se que somente $35 \%$ dos clones em estudo mostraram um percentual igual ou superior ao do IAN 873, este com $61 \%$ de plantas aptas a sangria.

A espessura de casca virgem aos sete anos de idade mostrou valores que variaram de $5,13 \mathrm{~mm}$ (IAC 324) a 7,37 mm (IAC 315), destacando-se ainda os clones IAC 319, IAC 328 e IAC 306 com 6,69 mm, $6,93 \mathrm{~mm}$ e $6,61 \mathrm{~mm}$, respectivamente, todos superiores ao IAN 873 que apresentou média de $6,44 \mathrm{~mm}$ (Tabela 5). A importância desse caracter consiste em influenciar indiretamente na produtividade do clone e proporcionar maior facilidade no procedimento de sangria (Gonçalves et al., 1994).

O número de anéis de vasos laticíferos contido na casca virgem, na abertura do painel, variou de 14,33 (IAC 324) a 29,77 anéis (IAC 315) (Tabela 5). Os clones IAC 315, IAC 328, IAC 306 e o IAC 323 apresentaram números médios de 29,77, 25,33, 24,60 e 24,00 anéis, respectivamente, todos superiores ao do IAN 873, que apresentou 23,40 anéis.

Quanto à resistência ao mal-das-folhas, destacaram-se os clones IAC 320, IAC 306, IAC 315, IAC 326, IAC 302, IAC 319 e IAC 300, igualando-se aos clones Fx 3864, Fx 3899 e IAN 873, pois apresentaram índices de doença dentro da classe II, ou seja, dentro da faixa de resistência (Tabela 6). $\mathrm{O}$ desfolhamento causado por M. ulei foi presenciado, em menor intensidade, nos clones IAN 873, Fx 3864, IAC 326, IAC 300, IAC 315, Fx 3899, IAC 306, IAC 302, IAC 320, IAN 6323 e IAC 319.

De uma forma geral, verifica-se que a doença aumentou na maioria dos clones a partir do ano de 1993, até o ano de 1994, passando a declinar a partir de 1995, chegando aos valores mínimos em 1996, com exceção do clone IAC 327 (Tabela 6). Este decréscimo dos níveis de doença pode ter ocorrido pelo fato de as plantas terem atingido a maturidade e por causa da entrada em senescência natural, em ciclos anuais, que influenciou negativamente a epidemia do mal-das-folhas, fenômeno denominado de "evitação" (Parleviliet, 1969; Pereira, 1988; Furtado, 1990; Menten, 1990). No litoral do Estado de São Paulo, plantas com hábito decíduo uniforme reenfolham num período com temperatura abaixo do ótimo para o patógeno, o que possibilita folhas sadias e livres de sintomas do mal-das-folhas. O clone IAC 327 tem como parentais clones de reconhecida produtividade, porém de alta suscetibilidade ao mal-das-folhas (RRIM 600), e apesar do hábito decíduo regular, sofre ataques epidêmicos de mal-das-folhas.

Através do acompanhamento do hábito da troca de folhas, no ano de 1996 (Tabela 6), verificou-se que a troca de folhas se iniciou na segunda quinzena de julho e se encerrou no início de outubro. Todos os clones testados, com exceção do clone Fx 3899, apresentaram o hábito regular de troca de folhas. Este hábito de troca de folhas é um aspecto bastante importante em programas de seleção de clones para 
TABELA 5. Classificação de caracteres relativos a abertura do painel de 20 clones de seringueira selecionados no Vale do Ribeira, Pariqüera-Açú, SP.

\begin{tabular}{|c|c|c|c|c|c|}
\hline Clones & $\begin{array}{l}\mathrm{N}^{\mathbf{o}} \mathrm{de} \\
\text { plantas }\end{array}$ & $\begin{array}{l}\text { Perímetro médio } \\
\text { do caule } \\
(\mathrm{cm})\end{array}$ & $\begin{array}{c}\text { Plantas aptas à } \\
\text { sangria }^{1} \\
(\%)\end{array}$ & $\begin{array}{l}\text { № médio de anéis de } \\
\text { vasos laticíferos }\end{array}$ & $\begin{array}{l}\text { Espessura média } \\
\text { de casca virgem } \\
(\mathrm{mm})\end{array}$ \\
\hline IAC 300 & 17 & 43,15 & 29 & 20,00 & 5,97 \\
\hline IAC 301 & 16 & 39,59 & 38 & 22,00 & 6,53 \\
\hline IAC 302 & 11 & 47,64 & 100 & 15,86 & 5,50 \\
\hline IAC 304 & 11 & 43,27 & 27 & 19,50 & 5,94 \\
\hline IAC 306 & 17 & 48,68 & 59 & 24,60 & 6,61 \\
\hline IAC 315 & 16 & 51,41 & 81 & 29,77 & 7,37 \\
\hline IAC 319 & 9 & 47,04 & 67 & 22,20 & 6,69 \\
\hline IAC 320 & 7 & 47,98 & 43 & 20,33 & 6,36 \\
\hline IAC 323 & 8 & 49,75 & 50 & 24,00 & 6,43 \\
\hline IAC 324 & 8 & 51,46 & 75 & 14,33 & 5,13 \\
\hline IAC 326 & 10 & 46,16 & 50 & 21,00 & 6,15 \\
\hline IAC 327 & 14 & 37,75 & 14 & 17,00 & 5,30 \\
\hline IAC 328 & 6 & 50,22 & 67 & 25,33 & 6,93 \\
\hline Fx 3864 & 10 & 52,20 & 90 & 17,78 & 5,45 \\
\hline Fx 3899 & 17 & 38,74 & 0 & - & - \\
\hline IAN 2903 & 10 & 38,45 & 10 & - & - \\
\hline IAN 3044 & 5 & 38,43 & 0 & 20,00 & 5,47 \\
\hline IAN 4493 & 15 & 37,40 & 7 & 21,00 & 5,62 \\
\hline IAN 6323 & 8 & 53,75 & 100 & 21,00 & 5,74 \\
\hline IAN $873^{2}$ & 51 & 47,99 & 61 & 23,40 & 6,44 \\
\hline Média & 13 & $45,50 \pm 5,46$ & & $21,06 \pm 3,66$ & $6,09 \pm 0,62$ \\
\hline
\end{tabular}

1 Árvores com perímetro superior a $45 \mathrm{~cm}$ a $1,20 \mathrm{~m}$ de altura do calo de enxertia.

2 Testemunha.

TABELA 6. Comportamento de clones quanto à incidência do mal-das-folhas, causado pelo fungo Microcyclus ulei, e hábito decíduo em 20 clones selecionados no Vale do Ribeira, Pariqüera-Açú, SP1.

\begin{tabular}{|c|c|c|c|c|c|c|c|}
\hline \multirow[t]{2}{*}{ Clones } & \multicolumn{5}{|c|}{ Índice de doença anual } & \multirow{2}{*}{$\begin{array}{c}\text { Classe de } \\
\text { doença }\end{array}$} & \multirow[t]{2}{*}{ Fenologia } \\
\hline & 1992 & 1993 & 1994 & 1995 & 1996 & & \\
\hline IAC 300 & 1,9 & 2,5 & 1,9 & 1,8 & 0,8 & $\mathrm{R}$ & Regular \\
\hline IAC 301 & 2,5 & 2,6 & 3,3 & 2,5 & 1,3 & MS & Regular \\
\hline IAC 302 & 1,4 & 2,6 & 3,3 & 1,3 & 0,0 & $\mathrm{R}$ & Regular \\
\hline IAC 304 & 2,7 & 3,1 & 3,5 & 2,7 & 0,8 & MS & Regular \\
\hline IAC 306 & 1,3 & 2,0 & 0,1 & 1,3 & 0,8 & $\mathrm{R}$ & Regular \\
\hline IAC 315 & 1,9 & 2,5 & 1,6 & 1,1 & 0,0 & $\mathrm{R}$ & Regular \\
\hline IAC 319 & 1,3 & 2,3 & 2,2 & 1,3 & 1,8 & $\mathrm{R}$ & Regular \\
\hline IAC 320 & 1,1 & 2,3 & 1,3 & 0,0 & 0,6 & $\mathrm{R}$ & Regular \\
\hline IAC 323 & na & na & na & na & na & & \\
\hline IAC 324 & na & na & na & na & na & & \\
\hline IAC 326 & 1,4 & 2,1 & 3,0 & 0,8 & 0,8 & $\mathrm{R}$ & Regular \\
\hline IAC 327 & 2,3 & 2,3 & 3,9 & 2,1 & 3,1 & MS & Regular \\
\hline IAC328 & na & $\mathrm{Na}$ & na & na & na & & \\
\hline Fx 3864 & 1,1 & 2,1 & 1,2 & 1,1 & 0,0 & $\mathrm{R}$ & Regular \\
\hline Fx 3899 & 1,3 & 2,0 & 0,7 & 1,1 & 0,0 & $\mathrm{R}$ & Irregular \\
\hline IAN 2903 & 1,1 & 2,4 & 3,5 & 1,7 & 0,2 & $\mathrm{R}$ & Regular \\
\hline IAN 3044 & 1,0 & 2,3 & 3,7 & 2,5 & 1,1 & MS & Regular \\
\hline IAN 4493 & 1,3 & 2,2 & 3,1 & 2,4 & 1,8 & MS & Regular \\
\hline IAN 6323 & 1,2 & 2,2 & 1,9 & 2,3 & 0,8 & $\mathrm{R}$ & Regular \\
\hline IAN 873 & 1,4 & 2,1 & 2,2 & 0,7 & 0,7 & $\mathrm{R}$ & Regular \\
\hline
\end{tabular}

10 a 1: AR (altamente resistente); 1,1 a 2: R (resistente); 2,1 a 3: MS (moderadamente suscetível); 3,1 a 4: AS (altamente suscetível); na: não avaliado. 
o controle do mal-das-folhas (Medeiros \& Bahia, 1971).

Dos clones utilizados no presente trabalho, o IAN 873 foi apontado por Furtado (1996) como o clone que apresentou a menor precocidade na troca de folhas, além de entrar em hibernação mais rápido que os demais. Os híbridos de $H$. benthamiana não deveriam ser recomendados para esta região, uma vez que eles perdem esta característica, como é o caso do Fx 3899.

\section{CONCLUSÕES}

1. Os clones IAN 6323, IAC 304 e Fx 3864 revelam melhor desempenho na média de produção de dois anos de sangria.

2. Em relação ao perímetro do caule, os clones IAC 315, IAC 328, Fx 3864, IAC 324, IAC 323 e IAN 6323 apresentam os melhores desempenhos.

3. Os clones IAC 320, IAC 306 e IAC 315 podem ser utilizados como fontes de resistência ao mal-dasfolhas em futuros trabalhos de melhoramento.

4. Os clones testados, com exceção do Fx 3899, apresentam hábito fenológico regular na troca de folhas.

\section{AGRADECIMENTOS}

Ao pesquisador Arlindo Pinheiro da Silveira, do Instituto Biológico, pelo auxílio na avaliação das doenças; à Andréa Cardoso Guerreiro e Lígia Regina Lima Gouvêa, do Instituto Agronômico, pelas análises do sistema laticífero contido na casca da seringueira; ao João Batista Vessalis e Ari de Camargo, do Núcleo de Agronomia do Vale do Ribeira e Núcleo Experimental de Campinas, respectivamente, pela coleta de dados no campo.

\section{REFERÊNCIAS}

ABRAHAM, P.D. Tapping of Hevea brasiliensis. In: SETHURAJ, M.R.; MATHEW, N.M. (Ed.). Natural rubber: biology, cultivation and technology. New York : Elsevier, 1992. p.263-281.

ABRAHAM, P.D.; TAYLER, R.S. Tapping of Hevea brasiliensis. Tropical Agriculture, St. Augustine, v.44, p.1-5, 1967.
CARDOSO, M. Instruções para a cultura da seringueira. Campinas : Instituto Agronômico, 1982. 43p. (IAC. Boletim, 196).

CHEE, K.H. Assessing susceptibility of Hevea clones to Microcyclus ulei. Annals of Applied Biology, Wellesbourne, v.84, n.2, p.135-145, 1976.

CUNHA, J.F. da. A seringueira (Hevea brasiliensis Muell. Arg.) no vale do rio Paraíba. Bragantia, Campinas, v.25, p.129-144, 1966.

EMBRAPA. Centro Nacional de Pesquisa de Seringueira e Dendê (Manaus, AM). Melhoramento genético da seringueira. Manaus, 1989. 23p. (EmbrapaCNPSD. Documentos, 10).

FURTADO, E.L. Comportamento decíduo da seringueira (Hevea spp.) e quantificação do mal das folhas causado por Microcyclus ulei (P. Henn.) v. Arx. Piracicaba : ESALQ, 1990. 82p. Dissertação de Mestrado.

FURTADO, E.L. Comportamento de cultivares de seringueira (Hevea spp.) frente ao mal das folhas da seringueira. Piracicaba : ESALQ, 1996. 79p. Tese de Doutorado.

GOMES, A.R.S.; VIRGENS FILHO, A.C.; MARQUES, J.R.B.; SANTOS, P.M. Avaliação de clones de seringueira (Hevea spp.) no sul da Bahia. In: SEMINÁRIO BRASILEIRO SOBRE RECOMENDAÇÃO DE CLONES DE SERINGUEIRA,1., 1982, Brasília. Anais. Brasília : SUDHEVEA, 1983. p.139-158.

GONÇALVES, P. de S. Melhoramento genético da seringueira (Hevea spp.) para o Estado de São Paulo. Campinas : IAC, 2000. 70p. Relatório de pesquisa apresentado à FAPESP.

GONÇALVES, P. de S.; CARDOSO, M.; BORTOLETTO, N. Redução do ciclo de melhoramento e seleção na obtenção de cultivares de seringueira. O Agronômico, Campinas, v.40, n.2, p.112130, 1988.

GONÇALVES, P. de S.; CARDOSO, M.; CAMPANA, M.; FURTADO, E.L.; TANZINI, M.R. Desempenho de novos clones de seringueira da série IAC. II. Seleções promissoras para a região do planalto do Estado de São Paulo. Pesquisa Agropecuária Brasileira, Brasília, v.29, n.8, p.1215-1224, ago. 1994.

MEDEIROS, A.G.; BAHIA, D.B. Estudos preliminares das enfermidades que causam a defolhação da seringueira, na Bahia (Brasil). Polímeros, Rio de Janeiro, v.1, n.2, p.9-18, 1971. 
MENTEN, J.O.M. Evitação: forma de defesa das plantas contra patógenos que deve ser melhor compreendida e explorada. Summa Phytopathologica, Piracicaba, v.16, n.2, p.77-83, 1990.

PAARDEKOOPER, E.C. Exploitation of the rubber tree. In: WEBSTER, C.C.; BAULKWILL, W.J. (Ed.). Rubber. New York : Longman 1989. p.349-414.

PARLEVILIET, J.E. Components of resistance that reduce the rate of epidemic development. Annual Review of Phytopathology, Palo Alto, v.17, p.203-222, 1969.

PEREIRA, R.E.A. Formas de defesa da seringueira (Hevea spp.) contra Microcyclus ulei (P. Henn.) v. Arx. Piracicaba : ESALQ, 1988. 67p. Dissertação de Mestrado.

SAKAI, E.; LEPSCH, I.F.; AMARAL, A.Z. do. Levantamento pedológico de reconhecimento semidetalhado da região do rio Ribeira do Iguape no Estado de São Paulo. I. Carta de solos da folha $24^{\circ} 15^{\prime}-24^{\circ} 45^{\prime} \mathrm{S}$ e $47^{\circ} 30^{\prime}-40^{\circ} \mathrm{W}$ (Registro, Juquiá, Pariqüera-Açú e Iguape). Campinas : Instituto Agronômico, 1983.

TAN, H. Strategies in rubber tree breeding. In: ABBOT, A.J.; ATKIN, R.K. (Ed.). Improving vegetatively propagated crops. London : Academic, 1987. p.2762.

WASTIE, R.L. Nursery screening of Hevea for resistance to Gloeosporium leaf disease. Rubber Research Institute of Malaysia Journal, Kuala Lumpur, v.23, p.339-350, 1973.

WYCHERLEY, P.R. Tapping and partition. Rubber Research Institute of Malaysia Journal, Kuala Lumpur, v.24, p.169-194, 1976. 